\title{
Splitting Magnitude Response into Real and Imaginary Parts
}

\author{
Vairis Shtrauss", Aldis Kalpinsh, and Uldis Lomanovskis \\ Institute for Material Mechanics, University of Latvia, Riga LV 1006, Latvia
}

\begin{abstract}
The determination of real and imaginary parts from magnitude responses is studied for causal linear time-invariant systems having monotonic impulse responses. It is demonstrated that the problem can be interpreted as a special filtering task in the Mellin transform domain having a diffuse magnitude response bounded by the magnitude responses of the filters corresponding to zero and maximum imaginary parts prescribed by the Kronig-Kramers relations. Discrete-time filters processing geometrically sampled magnitude responses are designed for determining the real and imaginary parts. Testing results are presented verifying the performance of the filters.
\end{abstract}

\section{Introduction}

Interconversion filters [1-3] operating with geometrically sampled data and executing an algorithm

$$
y\left(\omega_{0} q^{m}\right)=\sum_{n=-(N-1) / 2}^{(N-1) / 2} h[n] x\left(\omega_{0} q^{m-n}\right)
$$

are used for converting monotonic and locally monotonic material functions interrelated to each other by the Mellin convolution transform (MCT)

$$
y(\omega)=\int_{0}^{\infty} x(u) k\left(\frac{\omega}{u}\right) \frac{d u}{u},
$$

where $x($.$) is input function, y($.$) is output function, k($.$) is$ kernel, $h[n]$ is impulse response of filter (1), and $q>1$ is the common ratio for geometrically sampled data.

Interconversion filter (1) is related with the Mellin transform domain, where it has a periodic frequency response [1-3]

$$
H\left(e^{j \mu}\right)=\sum_{n=-(N-1) / 2}^{(N-1) / 2} h[n] \exp (-j \mu \ln q),
$$

which approximates the ideal frequency response, expressed by the Mellin transform of kernel $k(u)$

$$
H(j \mu)=M[k(u) ;-j \mu]=\int_{0}^{\infty} k(u) u^{-j \mu-1} d u
$$

where parameter $\mu$, named the Mellin frequency [2,3], is an equivalent of the angular frequency for signals on the logarithmic time domain.

Theoretical basis of the interconversion filtering [1-3] comes from the fact that MCT (2) for logarithmically transformed variables alters into the Fourier convolution type transform, which may be identified with an ideal interconversion filter operating on logarithmic time or frequency domain.

In this paper, we study the use of interconversion filters (1) for the determination of the real $J^{\prime}(\omega)$ and imaginary $J^{\prime \prime}(\omega)$ parts of the frequency response

$$
\widetilde{J}(\omega)=J^{\prime}(\omega)+j J^{\prime \prime}(\omega)
$$

from its magnitude response

$$
|\widetilde{J}(\omega)|=\sqrt{\left[J^{\prime}(\omega)\right]^{2}+\left[J^{\prime \prime}(\omega)\right]^{2}}
$$

for causal linear time-invariant systems (LTI) having monotonic impulse responses.

Although magnitude response (6) contains information about the real and imaginary parts, due to the operations of rising to the power and taking square root, neither the real part nor the imaginary part can be related to magnitude response (6) through MCT. Hence, converting the magnitude response into the real and imaginary parts does not represent a filtering task. On the other hand, the real and imaginary parts of frequency responses of causal physical systems are not wholly independent, but are linked by the Kramers-Kronig (KK) relations [4] ensuring the consistent magnitude responses. It points to a potent feasibility of converting the magnitude response into the real and imaginary parts. At present no methods known for the splitting the magnitude response into the real and imaginary parts. Nevertheless, development of such methods are of particular interest, because they may considerably simplify the determination of the real and imaginary parts, thanks to the fact that magnitude responses are relatively easily and accurately measurable signals, for example, by measuring the amplitudes of the steady-state responses to harmonic excitations [5-7].

Therefore, the final purpose of this study was to construct discrete-time transformers for converting magnitude response into the real part (further, $M R-R e$

\footnotetext{
$\bar{*}$ Corresponding author: strauss@pmi.lv
} 
transformer) and into the imaginary part (further, MR-Im transformer).

The rest of this paper is organized as follows. Theoretical background for converting the magnitude response into the real and imaginary parts is given in Section 2. Design of discrete-time MR-Re and MR-Im transformers are considered in Section 3. Testing results of the designed transformers are also presented here. Section 4 contains conclusions.

\section{Theoretical Background}

Two limiting cases may be considered for magnitude response (6): (i) - when the imaginary part is small and tends to zero $J^{\prime \prime}(\omega) \rightarrow 0$, and (ii) - when the imaginary part takes the maximum value $J^{\prime \prime}(\omega)=J_{\max }^{\prime \prime}(\omega)$.

\subsection{Limiting Case with Imaginary Part Tending to Zero}

In this case, the magnitude response approaches to the real part $|\widetilde{J}(\omega)| \rightarrow J^{\prime}(\omega)$ allowing to estimate the imaginary part by the KK relation [4]

$$
J^{\prime \prime}(\omega) \approx-\frac{2 \omega}{\pi} \int_{0}^{\infty} \frac{|\widetilde{J}(u)|}{u^{2}-\omega^{2}} d u,
$$

while the real part may be taken approximately equal to the magnitude response

$$
J^{\prime}(\omega) \approx|\widetilde{J}(\omega)| .
$$

As shown in [8], by dividing the numerator and denominator on $u^{2}$, KK relation (7) takes the MCT form (2) with $x(\omega)=|\widetilde{J}(\omega)|$ and $y(\omega)=J^{\prime \prime}(\omega)$, and kernel

$$
k^{\prime \prime}(u)=-2 u /\left(1-u^{2}\right) / \pi
$$

which according to (4) gives the frequency response [8]

$$
H_{\text {min }}^{\prime \prime}(j \mu)=M\left[k^{\prime \prime}(u) ; j \mu\right]=\tan (j \pi \mu / 2) \text {. }
$$

In its turn, Eq. (8) can be considered as the determination of $J^{\prime}(\omega)$ from $|\widetilde{J}(\omega)|$ by an all-pass filter with a constant (unity) frequency response

$$
H_{\min }^{\prime}(j \mu)=1 \text {. }
$$

\subsection{Limiting Case with Maximum Imaginary Part}

The phenomenological theories [9-11] state the following upper bound for the imaginary part of an elementary causal system with monotonic impulse response

$$
J_{\text {max }}^{\prime \prime}(\omega)=\frac{\Delta J \omega \tau}{1+\omega^{2} \tau^{2}}
$$

with the corresponding real part $\Delta J /\left(1+\omega^{2} \tau^{2}\right)$ according to $\mathrm{KK}$ relation $[4,8]$. Since frequency responses of real systems, such as materials [9-11], contain, as a rule, constant instantaneous values, we consider the real part with added constant $J_{\infty}$

$$
J_{\max }^{\prime}(\omega)=J_{\infty}+\frac{\Delta J}{1+\omega^{2} \tau^{2}}
$$

The parts (11) and (12) create magnitude response

$$
\begin{aligned}
\left|\widetilde{J}_{\max }(\omega)\right|=\sqrt{\left(J_{\infty}+\frac{\Delta J}{1+\omega^{2} \tau^{2}}\right)^{2}+\left(\frac{\Delta J \omega \tau}{1+\omega^{2} \tau^{2}}\right)^{2}}= \\
\sqrt{J_{\infty}^{2}+\frac{2 \Delta J J_{\infty}}{1+\omega^{2} \tau^{2}}+\frac{\Delta J^{2}}{1+\omega^{2} \tau^{2}}}
\end{aligned}
$$

where $\tau$ is characteristic time of the system.

Neither the real part (12) nor the imaginary part (11) can be related to the magnitude response (13) through MCT (2). We propose to approximate magnitude response (13) by a Mellin transformable function

$$
\begin{gathered}
A(\omega)=\sqrt{J_{\infty}^{2}+\frac{2 \Delta J J_{\infty}}{\sqrt{1+\omega^{2} \tau^{2}}}+\frac{\Delta J^{2}}{1+\omega^{2} \tau^{2}}}= \\
J_{\infty}+\frac{\Delta J}{\sqrt{1+\omega^{2} \tau^{2}}}
\end{gathered}
$$

which slightly overestimates magnitude response (13).

Contrary to the functions of pairs $\left\{J_{\text {max }}^{\prime}(\omega),|\widetilde{J}(\omega)|\right\}$ and $\left\{J_{\max }^{\prime \prime}(\omega),|\widetilde{J}(\omega)|\right\}$, the functions of pairs $\left\{J_{\text {max }}^{\prime}(\omega), A(\omega)\right\}$ and $\left\{J_{\text {max }}^{\prime \prime}(\omega), A(\omega)\right\}$ may be related to each other in the terms of the Mellin transforms

$$
\begin{aligned}
& M\left[J_{\max }^{\prime}(\omega) ;-j \mu\right]=M[A(\omega) ;-j \mu] \cdot H_{\max }^{\prime}(j \mu) ; \\
& M\left[J_{\max }^{\prime \prime}(\omega) ;-j \mu\right]=M[A(\omega) ;-j \mu] \cdot H_{\max }^{\prime \prime}(j \mu),
\end{aligned}
$$

where $H_{\max }^{\prime}(\mu)$ and $H_{\max }^{\prime \prime}(\mu)$ are frequency responses of MR-Re and MR-Im transformers in the limiting case with maximum imaginary part. According to (15) and (16), frequency responses $H_{\text {max }}^{\prime}(\mu)$ and $H_{\text {max }}^{\prime \prime}(\mu)$ may be determined as follows

$$
\begin{aligned}
& H_{\max }^{\prime}(j \mu)=M\left[J_{\max }^{\prime}(\omega) ;-j \mu\right] / M[A(j \mu) ;-j \mu] \\
& H_{\max }^{\prime \prime}(j \mu)=M\left[J_{\max }^{\prime \prime}(\omega) ;-j \mu\right] / M[A(j \mu) ;-j \mu] .
\end{aligned}
$$

By using the Mellin transforms

$$
\begin{gathered}
M\left[J_{\max }^{\prime}(\omega) ;-j \mu\right]=\frac{\pi}{2} \frac{\Delta J}{\sin (-j \pi \mu / 2)} ; \\
M\left[J_{\max }^{\prime \prime}(\omega) ;-j \mu\right]=\frac{\pi}{2} \frac{\Delta J}{\cos (j \pi \mu / 2)} ;
\end{gathered}
$$




$$
M[A(j \mu) ;-j \mu]=\frac{2^{-j \mu} \Gamma(2+j 2 \mu) \Gamma(-j \mu) \Delta J}{\Gamma(2+j \mu)},
$$

the following frequency responses were obtained for the MR-Re and MR-Im transformers in the limiting case with maximum imaginary part:

$$
\begin{aligned}
& H_{\max }^{\prime}(\mu)=\frac{\pi \Gamma(2+j \mu)}{2^{1-j \mu} \Gamma(2+j 2 \mu) \Gamma(-j \mu) \sin (-j \pi \mu / 2)} ; \\
& H_{\max }^{\prime \prime}(\mu)=\frac{\pi \Gamma(2+j \mu)}{2^{1-j \mu} \Gamma(2+j 2 \mu) \Gamma(-j \mu) \cos (j \pi \mu / 2)},
\end{aligned}
$$

where $\Gamma($.$) is gamma function.$

\subsection{Diffuse Magnitude Responses}

In Fig. 1, functions $\left|H_{\min }^{\prime}(\mu)\right|$ and $\left|H_{\text {max }}^{\prime}(\mu)\right|$ are shown, which form the bounds for the magnitude response of ideal MR-Re transformer. Hence, MR-Re transformer may be interpreted as an interconversion filter with a diffuse magnitude response with the constant - equal to (10) - lower bound and an increasing upper bound.

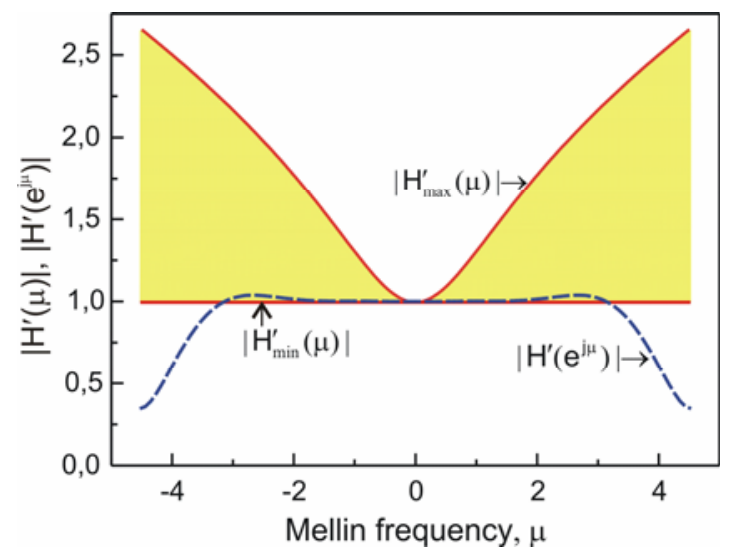

Fig. 1. The diffuse magnitude response of ideal MR-Re transformer (shaded area) and the magnitude response of 8-points discrete-time transformer (dashed).

In Fig. 2, functions $\left|H_{\min }^{\prime \prime}(\mu)\right|$ and $\left|H_{\max }^{\prime \prime}(\mu)\right|$ are shown, which also create the bounds of the diffuse magnitude response for ideal MR-Im transformer. In this case, the frequency response (9) establishes the lower bound, while the upper bound is again an increasing magnitude response with the shape likes to that of a differentiator of fractional order. At low Mellin frequencies $|\mu|<0.5$, MR-Im transformer has a united - not diffusing - magnitude response. This indicates actually on a practical feasibility of using KK transformers for computing the imaginary part from the magnitude response for narrow-band signals.

An important point is that MR-Im transformer has zero frequency response at zero frequency $H^{\prime \prime}(j 0)=0$, which ensures cutting out zero frequency component (DC component) of an input magnitude response and, thus, minimizing the effect of constant $J_{\infty}$.

\section{Design and Testing of Discrete-Time Transformers}

Discrete-time MR-Re and MR-Im transformers were designed by learning based on the system identification principle [1-3] to use pairs of the exact functions $\left\{J^{\prime}(\omega),|\widetilde{J}(\omega)|\right\}$ and $\left\{J^{\prime \prime}(\omega),|\widetilde{J}(\omega)|\right\}$ corresponding to the Cole-Cole model [12] with the width parameter 0.7. Based on the experience with discrete-time Kramers-Kronig transformers [8], we chose the common ratio $q=2$ and filter length $N=8$.

In Table, coefficients are given for the obtained discrete-time transformers. As seen the transformers are non-linear phases filters [13] having non-symmetric impulse responses. The appropriate magnitude responses are shown in Fig. 1 and Fig. 2 as dashed lines.

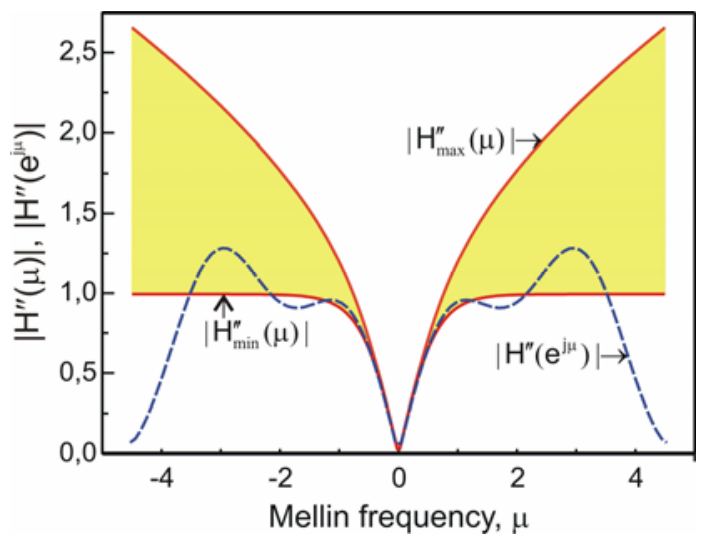

Fig. 2. The diffuse magnitude response of ideal MR-Im transformer (shaded area) and the magnitude response of 8 -points discrete-time transformer (dashed).

Table. Coefficients for MR-Re and MR-Im transformers

\begin{tabular}{|c|c|c|}
\hline \multirow{2}{*}{$\boldsymbol{n}$} & \multicolumn{2}{|c|}{ Coefficients $\boldsymbol{h}[\boldsymbol{n}]$} \\
\cline { 2 - 3 } & MR-Re transformer & MR-Im transformer \\
\hline-3.5 & -0.00862702 & 0.109042 \\
\hline-2.5 & 0.0474832 & 0.0134620 \\
\hline-1.5 & -0.143979 & 0.165268 \\
\hline-0.5 & 0.480793 & 0.601015 \\
\hline 0.5 & 0.759683 & -0.372957 \\
\hline 1.5 & -0.185825 & -0.470905 \\
\hline 2.5 & 0.0656697 & 0.136201 \\
\hline 3.5 & -0.0153284 & -0.178838 \\
\hline
\end{tabular}

To verify the performance, the transformers were tested by computing the real and imaginary parts of different shapes. We use the Havriliak-Negami model [14] with the following frequency response

$$
\widetilde{J}(\omega)=J_{\infty}+\Delta J\left[1+(j \omega \tau)^{\alpha}\right]^{\beta},
$$

which allows to change shapes of the real and imaginary parts, as well as of the magnitude responses within a wide range by varying parameters $\alpha, \beta, J_{\infty}$ and $\Delta J$.

In Figs. 3 and 4 , as examples, the exact real and imaginary parts (solid) and the computed ones (dashed) by the designed 8-points transformers are shown for $\beta=1$ and $\beta=0.5$, and five values of $\alpha$ to keep constant 
parameters $J_{\infty}=3, \Delta J=1, \tau=1$. The chosen values for the parameters $\alpha, \beta, J_{\infty}$ and $\Delta J$ are close to those used for describing complex dielectric permittivity of typical polymers $[9,10]$.

As seen, the accuracy of computing the real and imaginary parts is quite high, particularly, for the real parts at $\beta=0.5$, where it is hard to distinguish between the exact and computed curves.

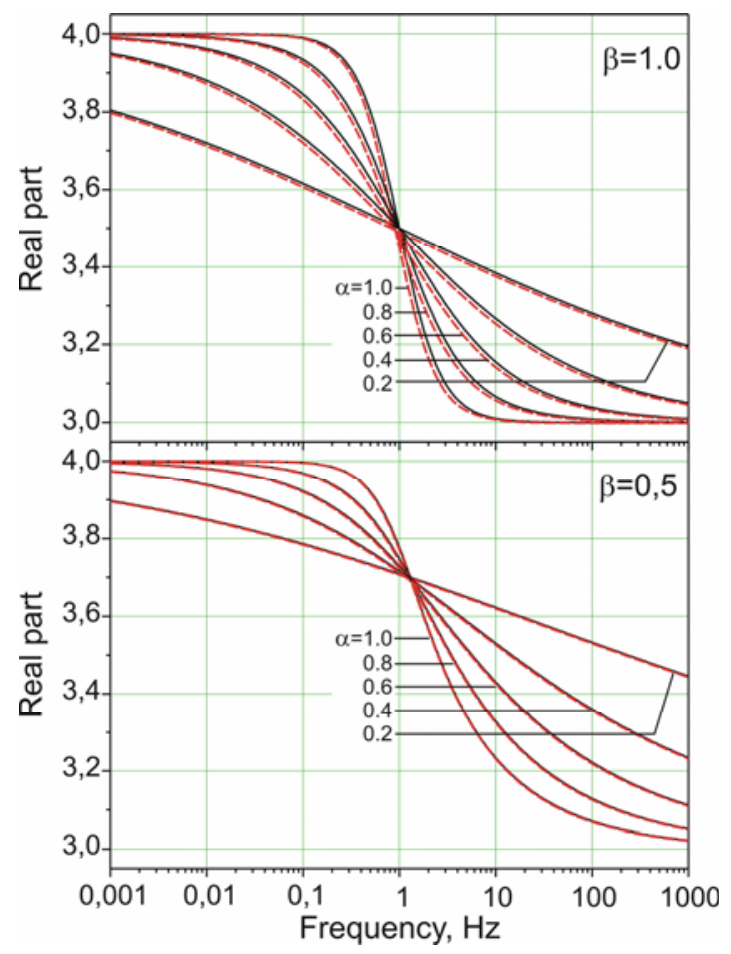

Fig. 3. Exact (solid) and computed (dashed) real parts.

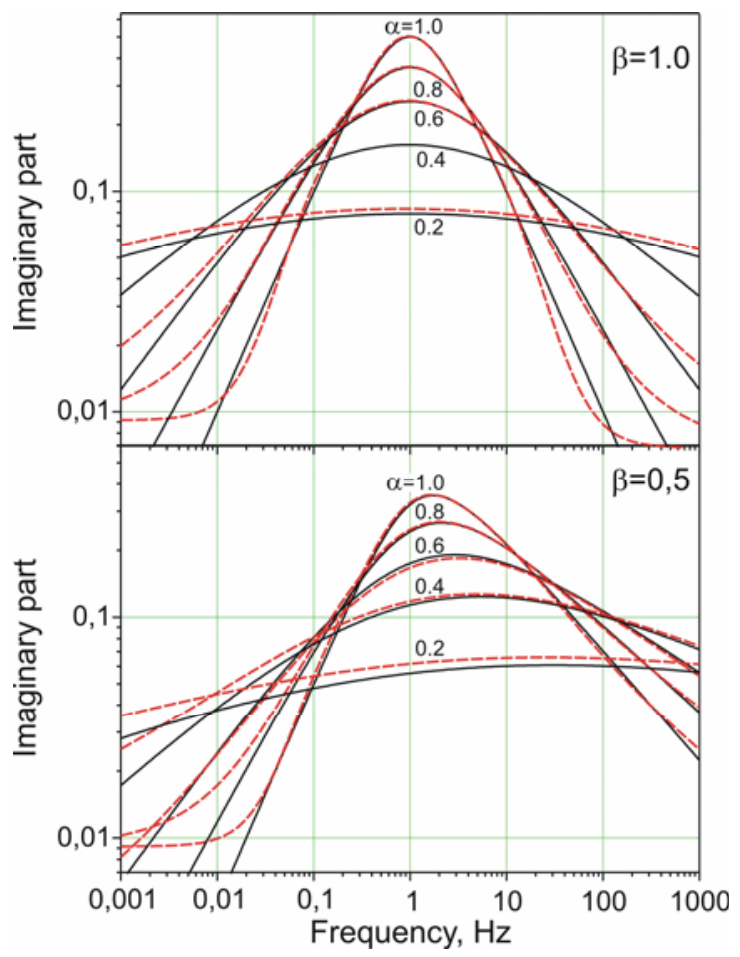

Fig. 4. Exact (solid) and computed (dashed) imaginary parts.

\section{Conclusions}

The use of interconversion filters [1-3], intended for transforming monotonic and locally monotonic material functions by executing the Mellin convolution transforms, is studied for the determination of the real and imaginary parts from the magnitude response for causal linear time-invariant systems having monotonic impulse responses. Since the problem cannot be described in the terms of a Mellin convolution, it does not represent an interconversion filtering task. It is demonstrated, however, that the problem in the limiting cases, when the imaginary part tends to zero and takes the maximum value, can be approximated by Mellin convolutions, which establishes the basis for interpretation the problem in the light of special interconversion filtering with diffuse frequency responses. The bounds of the diffuse frequency responses are found for determining the real and imaginary parts.

Discrete-time transformers are designed for converting the magnitude responses of causal linear time-invariant systems into the real and imaginary parts. Testing results of the transformers are presented.

This work was supported in part by the European Regional Development Fund under project No. 1.1.1.1/16/A/008.

\section{References}

1. V. Shtrauss, Signal Process. 45, 293 (1995).

2. V. Shtrauss, J. Non-Cryst. Solids 351, 2911 (2005).

3. V. Shtrauss, Digital Interconversion between Linear Rheologic and Viscoelastic Material Functions. In: Advances in Engineering Research 3, 91 (2012)

4. N. M. Nussenzveig, Causality and Dispersion Relations (1972)

5. V. Strauss, A. Kalpins, U. Lomanovskis, Patent WO2015047065A1, Method and device for estimating the real and imaginary parts of the frequency response (2013)

6. V. Shtrauss, A. Kalpinsh, WSEAS Trans. Appl. Theor. Mech. 7, 1, 29 (2012)

7. V. Shtrauss, A. Kalpinsh, Proc. 15th WSEAS International Conference on Systems. Recent Researches in System Science (CSCC '11) 106 (2011)

8. V. Shtrauss, Signal Process. 862887 (2006)

9. N. G. McCrum, B. E. Read, G. Williams, Anelastic and Dielectric Effects in Polymer Solids (1967)

10. A. K. Jonscher, Dielectric Relaxation in Solids (1983)

11. J. D. Ferry, Viscoelastic Properties of Polymers (1980)

12. K. S. Cole, R. H. Cole, J. Chem. Phys. 9, 341 (1941)

13. A. V. Oppenheim, R. V. Schafer, Discrete-Time Signal Processing (1999)

14. S. Havriliak, S. Negami, Polymer 8, 4, 161 (1967) 